\title{
Linearisation-based nonlinearity measures for wave-to-wire models in wave energy
}

\author{
Markel Penalba*, John V. Ringwood \\ Center for Ocean Energy Research, Maynooth University, Maynooth, Co. Kildare, Ireland
}

\section{A R T I C L E I N F O}

\section{Keywords:}

Wave energy

Nonlinearity measures

Best linear approximation

Wave-to-wire models

Power take-off dynamics

Energy maximising control

\begin{abstract}
A B S T R A C T
It is important to consider nonlinear effects when designing controllers to maximise generated energy in wave energy converters (WECs). Due to the substantial extra computation and complexity added when considering nonlinearities in the controller calculations, quantifying the extent of nonlinearity in WECs' behaviour is crucial to avoid designing overcomplicated control strategies. This paper suggests two nonlinearity measures to quantify the nonlinearity degree of wave-to-wire (W2W) models in steady-state, using the best linear approximation identified through a minimisation problem as a benchmark. The first measure, referred to as the original nonlinearity measure, evaluates the nonlinear effects of the wave-absorber hydrodynamic interaction. The second measure, referred to as the power nonlinearity measure, quantifies the nonlinear effects in power take-off (PTO) systems, considering the quadratic response of the power signal. The degree of nonlinearity of two WEC models, a partially-nonlinear hydrodynamic model with an ideal PTO model and a complete nonlinear W2W model, is evaluated using monochromatic and polychromatic waves over a wide range of wave periods and heights, covering the whole operational space of a WEC.
\end{abstract}

\section{Introduction}

Wave energy is one of the renewable energy sources that can substantially contribute to the future energy mix, due to the significant potential, up to $32000 \mathrm{TWh} /$ year (Mork et al., 2010), stored in ocean waves. Nevertheless, none of the suggested prototypes has yet shown economic viability.

Mathematical models that accurately describe the behaviour of wave energy converters (WECs) are crucial for the design, simulation and control of successful WECs. The energy maximising control problem can be particularly sensitive to model inaccuracies (Penalba et al., 2017d ), since a controller based on an imprecise model can even force the device to follow a trajectory that results in negative average absorbed power (Bacelli et al., 2015).

The vast majority of studies on energy maximising WEC control rely on linear hydrodynamic models originally developed for offshore engineering applications, which are based on boundary element method (BEM) codes that assume small motions of the devices around the mean position (Babarit and Delhommeau, 2015). However, the operation of WECs and other offshore structures, such as large platforms, is fundamentally different. While the majority of the offshore platforms are designed to remain still, WECs are designed to maximise energy absorption from ocean waves by encouraging device oscillations.
An alternative to BEM-based linear models are representative linear models (Giorgi, 2017), which have been shown to be more accurate than the BEM-based linear models, once the body motion becomes significant (Davidson et al., 2015a). However, these representative linear models only provide an average model over the full operational space (Davidson et al., 2015a, 2015b).

In addition, due to the extreme variability of the wave resource, WECs do not have specific operating points, meaning that standard setpoint following control strategies cannot be implemented. The absence of operating points also precludes the use of the normally useful linearisation techniques (Cheng et al., 2010). Therefore, the use of nonlinear models is somewhat inevitable in wave energy controller synthesis.

The sources of nonlinearities in wave energy systems are diverse, including wave-absorber hydrodynamic interactions (where the absorber is referred to as the part of the WEC that interacts directly with ocean waves) and power take-off (PTO) systems (Penalba et al., 2017a). These nonlinearities can be either lossy or lossless. Nonlinear FroudeKrylov (FK) forces, which are particularly relevant for point absorber (PA) WECs (Giorgi et al., 2016; Penalba et al., 2017d) are the main lossless nonlinear effects in wave-absorber hydrodynamic interactions, which can, in certain situations, lead to parametrically excited motion (Babarit et al., 2009; Tarrant and Meskell, 2016). With respect to the

\footnotetext{
* Corresponding author.

E-mail address: markel.penalbaretes.2015@mumail.ie (M. Penalba).
} 
PTO system, compressibility effects in hydraulic systems are the main lossless nonlinear effects.

Viscous effects are the primary lossy nonlinear effect in wave-absorber hydrodynamic interactions, which are especially relevant in oscillating surge wave converters (Giorgi et al., 2016). In addition, lossy nonlinearities are also important in hydraulic PTO systems, particularly when modelling the friction effects of the hydraulic cylinder and motor (Penalba et al., 2017b).

Some of these nonlinearities have already been considered in the design of nonlinear control strategies, as reviewed in (Faedo et al., 2017). For example, (Li, 2017), includes nonlinear static FK forces combining a pseudospectral method and differential flatness to efficiently tackle the nonlinear programming problem. Another study where nonlinear effects are considered in the controller is (Bacelli et al., 2015), where nonlinear viscous forces and non-ideal PTO efficiencies are incorporated. Hence, it is demonstrated that unless the controller is provided with precise information about the WEC behaviour, including accurate nonlinear effects, power production cannot be accurately maximised and constraint fulfilment cannot be ensured. In addition, nonlinear effects are exaggerated when implementing control, since WECs are encouraged to maximise their motion to optimise generated power (Giorgi et al., 2016).

However, including nonlinear effects in the controller significantly complicates the design of the controller. The first implication is the substantial increase in the numerical optimisation computation time, challenging real-time operation. In addition, the optimisation problem may become non-convex.

Therefore, identifying and quantifying nonlinear effects in WECs is crucial in accurately designing control strategies without overcomplicating the optimisation problem. For this purpose, a nonlinearity measure that quantifies the extent of nonlinear WEC behaviour, under different operational conditions, is vital.

The present paper presents two different nonlinearity measures to quantify nonlinear effects of the wave-absorber hydrodynamic interactions and PTO systems. The reminder of the paper is structured as follows: Different nonlinearity measures suggested in the literature are described in Section 2, the selected nonlinearity measure is briefly defined and its applicability for wave energy applications is analysed in Section 3, an adaptation of the selected nonlinearity measure is defined in Section 3.2, the case study and the results are presented in Sections 4 and 5 , respectively, and conclusions of the study are finally drawn in Section 6.

\section{A survey of nonlinearity measures}

The degree of nonlinearity of a system can be analysed through nonlinearity tests, evaluating the deviation of a property that linear systems hold. In this area, (Carter, 1987), describes coherence analysis and (Haber, 1985) presents a variety of methods, such as a frequency method analysing the presence of sub-harmonics, and methods based on linear correlation and linear spectral density.

Alternatively, a nonlinearity measure can be employed, which, unlike nonlinearity tests, provides a means to quantify the nonlinearity degree of a nonlinear system. Several approaches to evaluate nonlinearity of dynamic systems have been suggested in the literature. A review of these different approaches is presented in (Choudhury et al., 2008), where different methods to evaluate nonlinearity are divided into two main groups: time-series-based and model-based methods.

Time-series-based approaches only use the output time-series of a process to evaluate nonlinearity. Indeed, one of the main benefits of time-series-based approaches is that no identification of the process model is required. Three main time-series-based methods have been suggested: bicoherence-based (Emara-Shabaik et al., 1996; Choudhury et al., 2004), surrogate data-based (Theiler et al., 1992), and harmonic analysis approaches (Ruel and Gerry). Although time-series-based approaches only require the output data of the process to evaluate the nonlinearity of the system, this output data often needs to be adjusted (e.g. using correction factors that may include bias errors), or extended (e.g. generating synthetic or surrogate data).

Conversely, model-based approaches quantify the nonlinearity degree based on the input-output relationship of a nonlinear system. A curvature measure was first presented in (Guay et al., 1995) and extended in (Guay and McLellan, 1997), where the nonlinearity of a process is quantified by measuring the first and second order sensitivities of the process output signals. This sensitivity is measured by analysing the contribution of the higher-order terms of the Taylor expansion of a nonlinear operator, relative to the contribution of the firstorder terms. Thus, the curvature measure requires a Taylor expansion of the nonlinear system, which may be extremely complicated if the nonlinear system consists of different interconnected nonlinear subsystems, as is the case in the current application.

Other model-based approaches suggested in the literature measure the degree of nonlinearity by comparing the input-output behaviour of a nonlinear system to the best linear approximation of that nonlinear system. The first contribution to the identification of a best linear approximation is presented in (Desoer and Wang, 1980), and several authors have used the same definition since then. The need of nonlinear models for modelling and control applications is studied in (Nikolaou, 1993; Nikolaou and Hanagandi, 1994), where variations of the best linear approximation are evaluated for different inputs. Similarly, the best linear approximation for the worst input case is defined in (Allgöwer, 1995). A generalization of the approach suggested in (Allgöwer, 1995) is presented in (Helbig et al., 2000), which can be applied both to steady-state operation points and trajectory-dependent analyses.

A precise validated wave-to-wire (W2W) model, including all the different conversion stages from ocean waves to the electricity grid, is already available to analyse the input-output behaviour of a WEC (Penalba and Ringwood, 2019). Therefore, the model-based approach, suggested in (Helbig et al., 2000), appears to be a suitable measure to start with.

\section{Nonlinearity measures for wave energy system}

\subsection{Original measure definition}

Here we summarise the definition of the nonlinearity measure presented in (Helbig et al., 2000), which is a generic measure that can be used for any finite-dimensional multi-variable dynamic system. In the present paper, this nonlinearity measure is referred to as the original nonlinearity measure and is applied to WEC models, which can be described by a set of nonlinear ordinary differential equations.

Hence, a nonlinear WEC model can be represented by a state-space description as follows,

$\dot{\mathbf{x}}_{N}(t)=\mathbf{f}\left(\mathbf{x}_{N}(t), \mathbf{u}_{N}(t)\right), \quad \mathbf{x}_{N}(0)=\mathbf{x}_{N, 0}$,

$\mathbf{y}_{N}(t)=\mathbf{h}\left(\mathbf{x}_{N}(t), \mathbf{u}_{N}(t)\right), \quad 0 \leq t \leq t_{f}$

where $\mathbf{f}$ is a vector field, $\mathbf{h}$ a vector function, and $\mathbf{u}_{N}, \mathbf{x}_{N}, \mathbf{x}_{N, 0}$ and $\mathbf{y}_{N}$ are the inputs, states, initial conditions and outputs of the nonlinear system, respectively. The system can be evaluated for a finite or infinite time interval $\left(t_{f} \leq \infty\right)$. In this nonlinear system, $\mathbf{u}_{N}$ consists of elements of the space $\mathscr{U}_{a}$ of admissible inputs, $\mathbf{x}_{N}$ belongs to the space $\mathscr{X}, \mathbf{x}_{N, 0}$ are elements of the space of admissible initial conditions $\mathscr{X}_{0, a}$, and $\mathbf{y}_{N}$ consists of elements of a normed space of outputs $\mathscr{Y}$.

Let us define the nonlinear system presented in Equation (1) with a nonlinear dynamic operator that maps input signals $\mathbf{u}_{N}$ with $\mathbf{x}_{N, 0}$ initial conditions into output signals $\mathbf{y}_{N}$ as follows,

$\mathbf{y}_{N}=N\left[\mathbf{u}_{N}, \mathbf{x}_{N, 0}\right]$,

with $\mathbf{u}_{N} \in \mathscr{U}_{a}, \mathbf{x}_{N, 0} \in \mathscr{X}_{0, a}$, and $\mathbf{y}_{N} \in \mathscr{Y}$.

To quantify the nonlinearity of the system defined with the operator $N$, the best linear approximation needs to be identified for the same input 
and output dimensionality. As a consequence, a linear dynamic operator $L$ can be defined, where $L$ is an element of the time invariant linear space $\mathscr{L}$. Thus, any $L \in \mathscr{L}$ maps the input signals $\mathbf{u}_{L}$ with initial conditions $\mathbf{x}_{L, 0}$ into the output signals $\mathbf{y}_{L}$ as follows,

$\mathbf{y}_{L}=L\left[\mathbf{u}_{L}, \mathbf{x}_{L, 0}\right]$

where $\mathbf{u}_{L} \in \mathscr{U}_{L}, \mathbf{x}_{L, 0} \in \mathscr{X}_{0, L}, \mathbf{y}_{L} \in \mathscr{Y}_{L}, \mathscr{U}_{L}$ and $\mathscr{X}_{0, L}$ are the spaces of admissible inputs and initial conditions, respectively, and $\mathscr{Y}_{L}$ is the normed space of outputs.

In the present study, authors are only interested in measuring the nonlinearity of WECs' response at steady-state. Due to the slow variation of the conditions in the ocean, the transient-state can be neglected, as suggested in (Bacelli and Ringwood, 2015; Faedo et al., 2018a) for energy maximisation control applications. Therefore, zero initial conditions (ZICs) can be assumed $\left(\mathbf{x}_{N, 0}=\mathbf{x}_{L, 0}=0\right)$.

Once the nonlinear and linear systems are defined, the original nonlinearity measure is represented as follows,

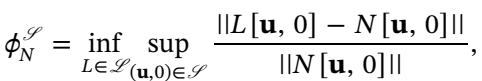

with

$S=\left\{(\mathbf{u}, 0): \mathbf{u} \in \mathscr{U}_{a}, N[\mathbf{u}, 0] \in \mathscr{Y}_{a}\right\}$,

where $\mathbf{u}$ refers to the common inputs for the linear and the nonlinear systems, $\mathscr{Y}_{a} \subset \mathscr{Y}$ is the space of admissible outputs, and $\|\cdot\|$ is a suitable norm. The nonlinearity measure in Equation (4) assumes the existence of $\mathbf{y}$ and the boundedness of all norms for all $(\mathbf{u}, 0) \in \mathscr{S}$.

The nonlinearity degree of a nonlinear system is given by the normalized largest difference between the nonlinear system and the best linear approximation for the worst input. This nonlinearity measure always provides a value between 0 and 1 , where 0 means that $N$ behaves as a linear system, and the closer to 1 , the more nonlinear $N$ is. A suitable norm applied in Equation (4) is the $\mathrm{L}_{2}$-norm.

The computation of the nonlinearity measure presented in Equation (4) requires the solution of a practically infeasible infinite-dimensional nonlinear min-max problem. Therefore, Equation (4) needs to be transformed into a finite-dimensional optimisation problem, replacing the inf-operator in Equation (4) with a min-operator. To that end, it is necessary to represent the best linear approximation $L$ as a weighted sum of basis functions $L_{i}$ as follows,

$L\left[u_{j}, 0\right]=\sum_{i=1}^{m} d_{w, i} L_{i}\left[u_{j}, 0\right]$,

where $\mathbf{d}_{\mathbf{w}}=\left[d_{w, 1}, d_{w, 2}, \ldots, d_{w, m}\right] \in \mathscr{R}^{m}$ is the vector of weights. The space spanned by the $L_{i}$ basis functions should be dense in $\mathscr{L}$. Following (Helbig et al., 2000), the linear operator $L$ is represented by linear transfer functions $L_{i}$, with corresponding time constants $T_{i}$,

$L_{i}(s)=\frac{1}{1+T_{i} s}$.

In addition, if an approximated space $\mathscr{S}_{c} \subset \mathscr{S}$ with a finite number of elements is defined, the sup-operator in Equation (4) can be replaced by a max-operator. Hence, the inf-sup problem becomes a min-max optimisation problem.

Finally, the min-max optimisation problem is transformed into a single convex minimisation problem with constraints:

$\theta_{N}^{\mathscr{S}_{c}}=\min _{z \in \mathscr{R}, \mathbf{d} \in \mathscr{R} m} z$,

s. t. $\frac{L\left[u_{j}, 0\right]-N\left[u_{j}, 0\right]}{N\left[u_{j}, 0\right]}-z \leq 0$,

$\forall\left(u_{j}, 0\right) \in \mathscr{S}_{c}$,

Once the finite dimensional problem is defined as a single convex minimisation problem, this can be solved using state of the art optimisation solvers, such as those detailed in (Boyd and Vandenberghe, 2004). For further details about the transformation of the optimisation

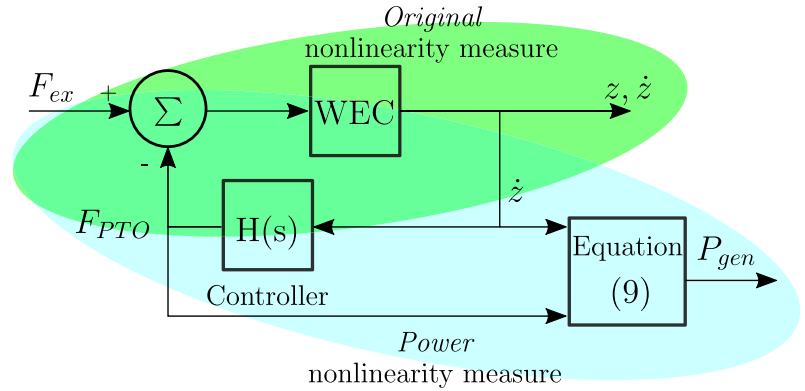

Fig. 1. Diagram of a WEC, illustrating the applicability of the original and power nonlinearity measures.

formulation of Equation (4) into Equation (8), the reader is referred to (Helbig et al., 2000).

\subsection{A power nonlinearity measure}

In wave energy systems, excitation force $\left(F_{e x}\right)$ is the typical input $\left(\mathbf{u}_{N}\right)$ for WECs, while the output $\left(\mathbf{y}_{N}\right)$ is typically position (z), velocity $(\dot{z})$ and/or generated power $\left(P_{\text {gen }}\right)$, as illustrated in Fig. 1.

The original nonlinearity measure can quantify nonlinearities of the WEC system when using $z$ or $\dot{z}$ as outputs. That way, $\theta$ can capture any nonlinearity in the hydrodynamic model. However, generated power is given as follows,

$P_{\text {gen }}=-\dot{z} F_{P T O}$,

where

$F_{P T O}=-z K_{P T O}-\dot{z} B_{P T O}=f(z, \dot{z})$,

and $B_{\text {PтO }}$ and $K_{\text {РтO }}$ are the damping and the stiffness of the PTO model.

Hence, generated power is represented by a nonlinear combination of the outputs, $P_{\text {gen }}=f\left(z \cdot \dot{z}, \dot{z}^{2}\right)$. Using $P_{\text {gen }}$ as output, $\theta$ results biased by the nonlinearities in the measurement and cannot provide the nonlinearity degree of the WEC system. However, the nonlinearity degree of a WEC, including all the dynamics from waves to the electricity grid, can only be assessed using $P_{\text {gen }}$ as output. Therefore, for the nonlinearity measure to be used for evaluating the nonlinearity degree of W2W models, $\theta$ must be adapted. The adaptation of $\theta$ is referred to as the power nonlinearity measure in the following. Fig. 1 illustrates the application area of the original and power nonlinearity measures.

Since $P_{\text {gen }}$ is obtained by multiplying $\dot{z}$ and $F_{P T O}$, the simplest way to measure the nonlinearity of a system using $P_{\text {gen }}$ as output is including that multiplication between $\dot{z}$ and $F_{\text {РТО }}$ in the nonlinearity measure.

Thus, two linear operators $L_{1}$ and $L_{2}$, which represent $\dot{z}$ and $F_{\text {РTO, }}$, can be defined so that the combination of any $L_{1} \in \mathscr{L}$ and any $L_{2} \in \mathscr{L}$ maps the input signals $\mathbf{u}_{L_{12}}$, with initial conditions $\mathbf{x}_{L_{1}, 0}$ and $\mathbf{x}_{L_{2}, 0}$ into the output signals $\mathbf{y}_{L_{12}}$ as follows,

$\mathbf{y}_{L_{12}}=L_{1}\left[\mathbf{u}_{L_{12}}, \mathbf{x}_{L_{1}, 0}\right] L_{2}\left[\mathbf{u}_{L_{12}}, \mathbf{x}_{L_{2}, 0}\right]$,

with $\mathbf{u}_{L_{12}} \in \mathscr{U}_{L_{12}}, \mathbf{x}_{L_{1}, 0}, \mathbf{x}_{L_{2}, 0} \in \mathscr{X}_{0, L_{12}}$, and $\mathbf{y}_{L_{12}} \in \mathscr{Y}_{L_{12}}$.

The new best linear system is then formed by the combination of two linear operators $\left(L_{1}[\mathbf{u}, 0] L_{2}[\mathbf{u}, 0]\right)$, so the power nonlinearity measure can be defined as follows,

$\psi_{N}^{\mathscr{S}_{p}}=\inf _{L_{1}, L_{2} \in \mathscr{L}_{(\mathbf{u}, 0) \in \mathscr{S}_{p}}} \frac{\left\|L_{1}[\mathbf{u}, 0] L_{2}[\mathbf{u}, 0]-N[\mathbf{u}, 0]\right\|}{\|N[\mathbf{u}, 0]\|}$

with

$S_{p}=\left\{(\mathbf{u}, 0): \mathbf{u} \in \mathscr{U}_{a}, N[\mathbf{u}, 0] \in \mathscr{Y}_{p}\right\}$

where $L_{1}[\mathbf{u}, 0]: \mathscr{U}_{a} \times 0 \rightarrow \mathscr{Y}_{a}$ and $L_{2}[\mathbf{u}, 0]: \mathscr{U}_{a} \times 0 \rightarrow \mathscr{Y}_{a}$ are two linear dynamic operators belonging to the space of linear operators $\mathscr{L}$. Similarly to the measure presented in Equation (4), $\mathscr{U}_{a}$ and $\mathscr{Y}_{a}$ are the spaces of admissible inputs and outputs of the two linear operators, 
respectively. However, the combination of these two linear operators defines a new space of admissible outputs $\mathscr{Y}_{p}$. In addition, The nonlinearity measure in Equation (12) assumes the existence of $\mathbf{y}$ and the boundedness of all norms for all $(\mathbf{u}, 0) \in \mathscr{S}$.

Similarly to the measure presented in Equation (4), the computation of the power nonlinearity measure needs an approximative computation scheme, transforming the practically infeasible problem, into a finite dimensional optimisation problem. Following Equation (14), with the replacement of the inf- and sup-operators with min- and max-operators, respectively, the power nonlinearity measure results in a single constrained minimisation problem as follows,

$\chi_{N}^{\mathscr{S}^{p c}}=\min _{z \in \mathscr{R}, \mathbf{d}_{1}, \mathbf{d}_{2} \in \mathscr{R}^{m}} z$

s. t. $\frac{L_{1}\left[u_{j}, 0\right] L_{2}\left[u_{j}, 0\right]-N\left[u_{j}, 0\right]}{N\left[u_{j}, 0\right]}-z \leq 0$

$\forall\left(u_{j}, 0\right) \in \mathscr{S}_{p c}$

where $S_{p c} \subset \mathscr{S}_{p}$ is the approximated space with a finite number of elements, and $\mathbf{d}_{1}$ and $\mathbf{d}_{2}$ vectors of weights of $L_{1}$ and $L_{2}$, respectively. For further details about the final transformation, the reader is referred to (Helbig et al., 2000).

That way, the best linear approximation obtained from the power nonlinearity measure suggested in Equation (12) captures the quadratic response of the power signal, and is able to provide the nonlinearity measure expected for a linear model $(\chi=0)$.

It is important to note that this coverage of the quadratic response does not distort the nonlinearity measure, since it only covers the quadratic response corresponding to power. Other nonlinearity sources are still captured by the power nonlinearity measure (see Section 5).

\section{Case study}

The nonlinearity measures described in Sections 3 and 3.2 are applied to assess the nonlinearity of the behaviour of a spherical heaving PA of $5 \mathrm{~m}$ diameter. This spherical PA is of particular interest due to its non-uniform cross-sectional area, which leads to nonlinear FK forces (Penalba et al., 2017d ). The natural period of this spherical PA is 3.17s, is assumed to be deployed in deep-water, and is restricted to heave motion only to simplify the analysis. Fig. 2 illustrates the diagram of the WEC.

In the present paper, two models that include different nonlinear effects are considered. On the one hand, a partially-nonlinear hydrodynamic model, referred to as NLHyd model in the following, is

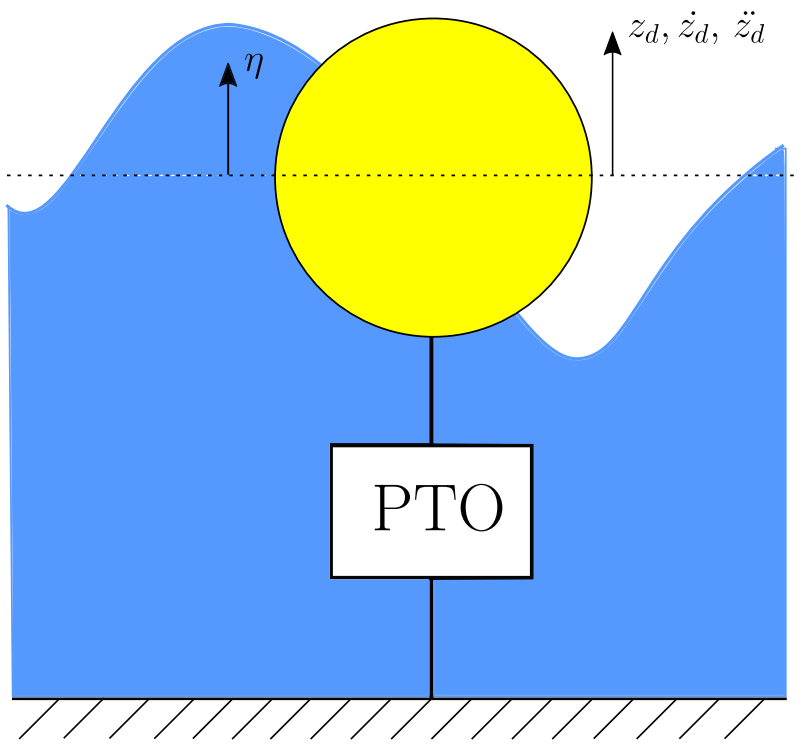

Fig. 2. Illustrative diagram of the spherical heaving PA. analysed. The NLHyd model includes nonlinear FK forces and viscous effects in the wave-absorber hydrodynamic interaction as follows,

$$
\begin{aligned}
M \ddot{z}_{d}(t)= & F_{F K}(t)+\int_{-\infty}^{\infty} K_{d i f f}(t-\tau) \eta(\tau) d \tau-\mu_{\infty} \ddot{z}_{d}(t) \\
& -\int_{-\infty}^{\infty} K_{\text {rad }}(t-\tau) \dot{z}_{d}(\tau) d \tau+F_{P T O}+F_{v i s c},
\end{aligned}
$$

where $z_{d}, \dot{z}_{d}$ and $\ddot{z}_{d}$ are the displacement, velocity and acceleration of the WEC, respectively, $M$ is the mass of the WEC, $K_{\text {diff }}$ the diffraction impulse response function (IRF), $\eta$ the free-surface elevation, $\mu_{\infty}$ the added-mass at infinite frequency, $K_{\text {rad }}$ the radiation IRF.

Nonlinear FK forces and viscous effects are included using the algebraic solution of the integral presented in (Giorgi and Ringwood, 2016a) and a Morison-like equation (Morison et al., ), respectively,

$F_{F K}=F_{g}-2 \pi \rho g\left[\frac{\sigma^{3}}{3}+z_{d} \frac{\sigma^{2}}{2}\right]_{\sigma_{1}}^{\sigma_{2}}-\frac{2 \pi}{\chi} \rho g a \cos (\omega t)\left[\left(z_{d}+\frac{1}{\chi}-v\right) e^{\chi \sigma}\right]_{\sigma_{1}}^{\sigma_{2}}$,

$F_{\text {visc }}=-\frac{1}{2} \rho C_{d} A_{d}(t)\left|\dot{z}_{d}-\dot{\eta}\right|\left(\dot{z}_{d}-\dot{\eta}\right)$,

where $F_{g}$ is the gravity force, $\rho$ the water density, $g$ the acceleration due to the gravity, $a$ the wave amplitude, $\omega$ the wave frequency, $\chi$ the wavenumber, $C_{d}$ the drag coefficient, $A_{d}$ the instantaneous cross-sectional area of the device, and $\dot{\eta}$ the velocity of the undisturbed wave field. Integration limits $\sigma_{1}=z-H$ and $\sigma_{2}=\eta$ define the instantaneous wetted surface, $H$ is the draft of the device, $\sigma$ and $\theta$ are the parametric cylindrical coordinates of the algebraic solution, and $x$ and $z$ the Cartesian coordinates. The PTO system in the NLHyd model is represented by Equations (10) and (9).

On the other hand, a complete W2W model, henceforth referred to as NLW2W model, is considered. The $N L W 2 W$ model combines the hydrodynamic model incorporated in the NLHyd model with a highfidelity PTO model, where all the necessary nonlinear dynamics and losses are included (Penalba and Ringwood, 2019). The PTO force in the $N L W 2 W$ model, including compressibility effects and friction losses $\left(F_{\text {fric }}\right)$, is calculated using Equations 18-20, rather than Equation (10).

$F_{\text {PTO }}=A_{p} \Delta p+F_{\text {fric }}$,

$\dot{p}=\frac{\beta_{e f f}(p)}{V+A_{p} x_{p}}\left(Q-\dot{x}_{p} A_{p}\right)$,

$F_{\text {fric }}=\sigma_{v} \dot{x}_{p}+\operatorname{sign}\left(\dot{x}_{p}\right)\left[F_{c}+F_{s t} \exp \left(-\frac{\left|\dot{x}_{p}\right|}{c_{s t}}\right)\right]$,

where $A_{p}$ is the piston area, $p$ the pressure in each chamber of the hydraulic cylinder, $\Delta p$ the pressure difference between the chambers, $x_{p}$ and $\dot{x}_{p}$ the piston position and velocity, $Q$ is the flow entering or exiting the cylinder chamber, $\beta_{\text {eff }}(p)$ the effective bulk modulus and $V$ the minimum volume (calculated when the piston reaches its minimum or maximum position) in the cylinder chamber. Friction in the cylinder is included using the Stribeck model (Jelali and Kroll, 2012), for which $\sigma_{v}$ is the viscous coefficient, $F_{c}$ the Coulomb friction force, $F_{s t}$ the static friction force, and $c_{s t}$ the characteristic velocity of the Stribeck curve.

The hydraulic motor, also represented in the NLW2W model, including volumetric and mechanical losses via the Schlösser loss model (Schlöesser, 1961; Schlösser, 1968), is modelled as follows,

$Q_{M}=\alpha D_{\omega} \omega_{M}-\Delta p_{M} C_{Q 1}$,

$T_{M}=\alpha D_{\omega} \Delta p_{M}-\left(C_{T 1}+C_{T 2} \Delta p_{M}+C_{T 3} \omega_{M}+C_{T 4} \omega_{M}^{2}\right)$,

where $\alpha$ is the motor displacement fraction, $D_{\omega}$ the displacement of the hydraulic motor, $\omega_{M}$ the rotational speed of the shaft, $\Delta p_{M}$ the pressure difference across the hydraulic motor, and $C_{Q 1}, C_{T 1}, C_{T 2}, C_{T 3}$ and $C_{T 4}$ the parameters of the Schösser loss model.

Finally, the squirrel-cage induction generator in the $N L W 2 W$ model is incorporated using the equivalent two-phase $(d q)$ equations (Krause 


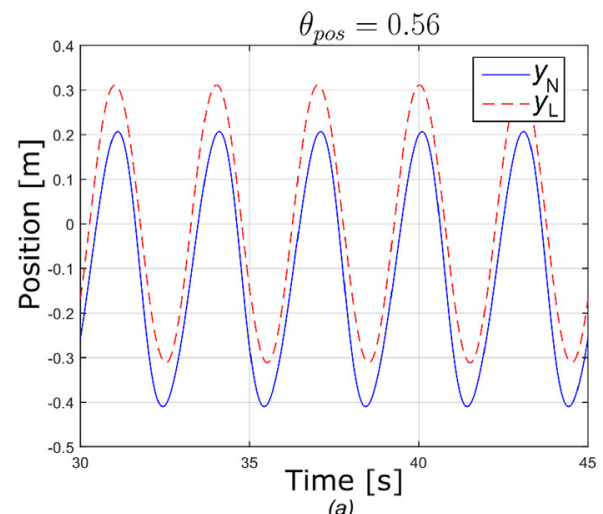

(a)

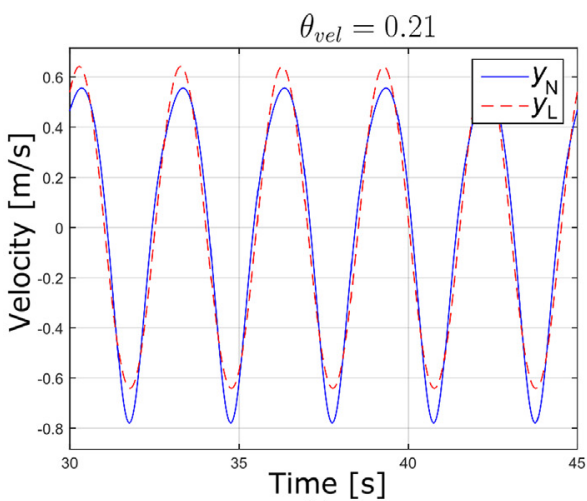

(b)

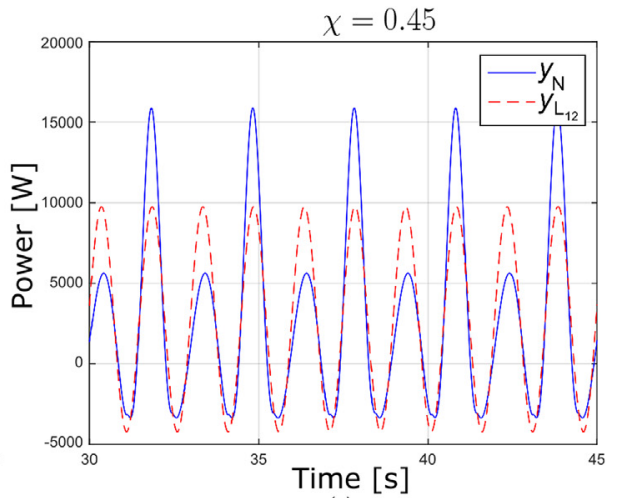

(c)

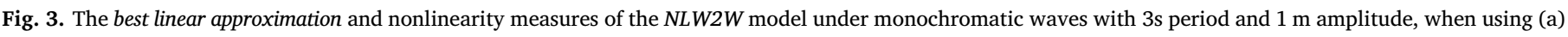
position, (b) velocity and (c) power.

et al., 2013) as follows,

$V_{s d}=R_{s} i_{s d}-\omega \lambda_{s q}+L_{s} \frac{d}{d t} i_{s d}+L_{\mathrm{m}} \frac{d}{d t}\left(i_{s d}+i_{r d}\right)$,

$V_{s q}=R_{s} i_{s q}+\omega \lambda_{s d}+L_{s} \frac{d}{d t} i_{s q}+L_{\mathrm{m}} \frac{d}{d t}\left(i_{s q}+i_{r q}\right)$,

$0=R_{r} i_{r d}-\left(\omega-\omega_{r}\right) \lambda_{r q}+L_{r} \frac{d}{d t} i_{r d}+L_{\mathrm{m}} \frac{d}{d t}\left(i_{s d}+i_{r d}\right)$,

$0=R_{r} i_{r q}+\left(\omega-\omega_{r}\right) \lambda_{r d}+L_{r} \frac{d}{d t} i_{r q}+L_{\mathrm{m}} \frac{d}{d t}\left(i_{s q}+i_{r q}\right)$,

where $V$ is the voltage, $i$ the current, $R$ the resistance and $\lambda$ the flux. Subscripts $s$ and $r$ are used for the stator and rotor, while $d$ and $q$ refer to the direct and quadrature axes, respectively. $\omega$ and $\omega_{r}$ are the angular speed of the reference frame and the rotor, respectively, and flux linkage expressions are given as,

$\lambda_{s d}=\left(L_{s}+L_{\mathrm{m}}\right) i_{s d}+L_{\mathrm{m}} i_{r d}$,

$\lambda_{s q}=\left(L_{s}+L_{\mathrm{m}}\right) i_{s q}+L_{\mathrm{m}} i_{r q}$,

$\lambda_{r d}=\left(L_{r}+L_{\mathrm{m}}\right) i_{r d}+L_{m} i_{s d}$,

$\lambda_{r q}=\left(L_{r}+L_{\mathrm{m}}\right) i_{r q}+L_{\mathrm{m}} i_{s q}$

where $L_{s}$ and $L_{r}$ are the stator and rotor leakage inductances, respectively, and $L_{m}$ is the mutual inductance. Flux linkages, voltages and currents allow for the calculation of the electromagnetic torque $\left(T_{e}\right)$, rotational speed of the rotor and generated electrical power can be calculated,

$T_{e}=\frac{3 N_{p}}{4}\left(\lambda_{s d} i_{s q}-\lambda_{s q} i_{s d}\right)$,

$\dot{\omega}_{r}=\frac{N_{p}}{2 J}\left(T_{e}-T_{M}-B_{\text {wind }} \omega_{r}\right)$,

$P_{g e n}=\frac{3}{2}\left(V_{s d} i_{s d}+V_{s q} i_{s q}\right)$

where $N_{p}$ is the number of poles in the generator and $B_{\text {wind }}$ the friction/ windage damping.

To asses the representative nonlinearity of a model, it is crucial to carefully cover the operational space of the WEC. To that end, sea-states (wave periods and heights) and implemented control strategies need to be selected. Based on the wave resource in the Biscay Marine Energy Platform (BIMEP) test site, located in the Bay of Biscay (Penalba et al., $2017 \mathrm{c}$ ), wave periods from $3 \mathrm{~s}$ to $12 \mathrm{~s}$ and wave heights from $1 \mathrm{~m}$ to $5 \mathrm{~m}$ are analysed. Although real ocean waves are polychromatic waves, the additional analysis for monochromatic waves provides insight into the response of the WEC at each exciting frequency. Therefore, both monochromatic and polychromatic waves are considered.
With respect to the control strategies, the nonlinearity degree of the spherical WEC is assessed under a docile and a more aggressive control strategy, resistive (ResC) and reactive control (ReacC), respectively. The two control strategies are implemented using the control parameters $B_{P T O}$ and $K_{P T O}$ in Equation (10), with $K_{P T O}=0$ in the ResC case. Optimal control parameters for each sea-state are used, from (Penalba and Ringwood, 2018).

\section{Results}

The nonlinear behaviour of the spherical WEC is first assessed with monochromatic waves. In this case, the best linear approximation is parametrized by 2 basis functions, using two time constants $T_{1}=\frac{2 \pi}{0.3} S$ and $T_{2}=\frac{2 \pi}{2} S$. These time constants are selected to accurately characterize the steady-state response of the device over the whole frequency range, as demonstrated in (Faedo et al., 2018b), with one time constant close to the resonant frequency $\left(T_{2}\right)$ and the second time constant at a lower frequency $\left(T_{1}\right)$.

Before evaluating the nonlinear behaviour of the WEC over the whole operational space, it should be noted that different values of the nonlinearity measure can be obtained for the same model and operational conditions (identical input and control), depending on the output signal selected for the evaluation. Fig. 3 (a), (b) and (c) illustrate the best linear approximation and the nonlinearity measure of the NLW2W model, when respectively using position $\left(\theta_{p o s}\right)$, velocity $\left(\theta_{v e l}\right)$ and power $(\chi)$ to assess the nonlinearity degree under monochromatic waves (3s period and $1 \mathrm{~m}$ height, close to resonance). The highest value of the nonlinearity measure is obtained with position as output $\left(\theta_{\text {pos }}=0.56\right)$, higher than with power $(\chi=0.45)$, which includes all the nonlinearities of the high-fidelity PTO model described in Equations 18-33. In addition, the nonlinearity degree is more than halved using velocity as output compared to $\theta_{\text {pos }}\left(\theta_{\text {vel }}=0.21\right)$.

This substantial difference between $\theta_{\text {pos }}$ and $\theta_{\text {vel }}$ appears due to the specific nature of the nonlinearity in this particular case. The main nonlinear phenomenon of the position signal is the asymmetry with respect to the $\mathrm{x}$-axis at the origin, which can be represented by a constant offset in steady-state. However, because velocity is the derivative of the position, the offset of the position vanishes in the derivation, dramatically reducing the nonlinear behaviour.

The relevance of nonlinearities in different outputs depends on the application of the nonlinear model. When nonlinearity is assessed for energy maximising control applications in wave energy, the implication of velocity nonlinearities may be significantly more influential than position nonlinearities for two main reasons: On the one hand, velocity components are lossy, which strongly affects the final energy generation, and, on the other hand, the control problem in wave energy is commonly tackled optimizing the velocity trajectory, as reviewed in 


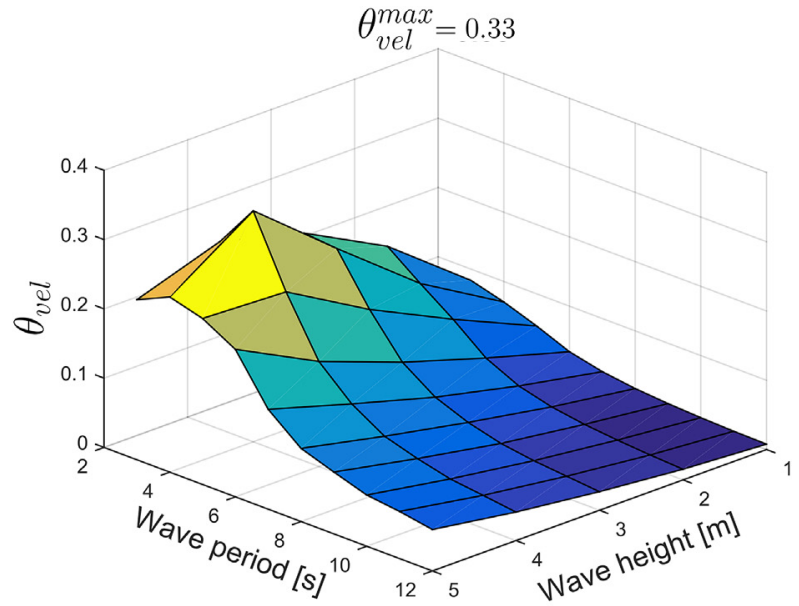

(a)

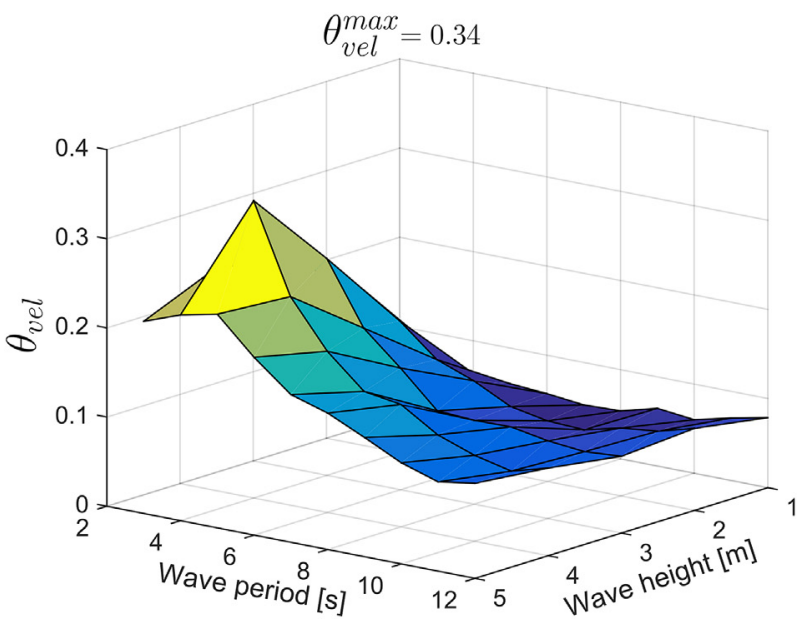

(b)

Figure 4. $\theta_{\text {vel }}$ over the whole operational space for the NLHyd model under (a) ResC and (b) ReacC.

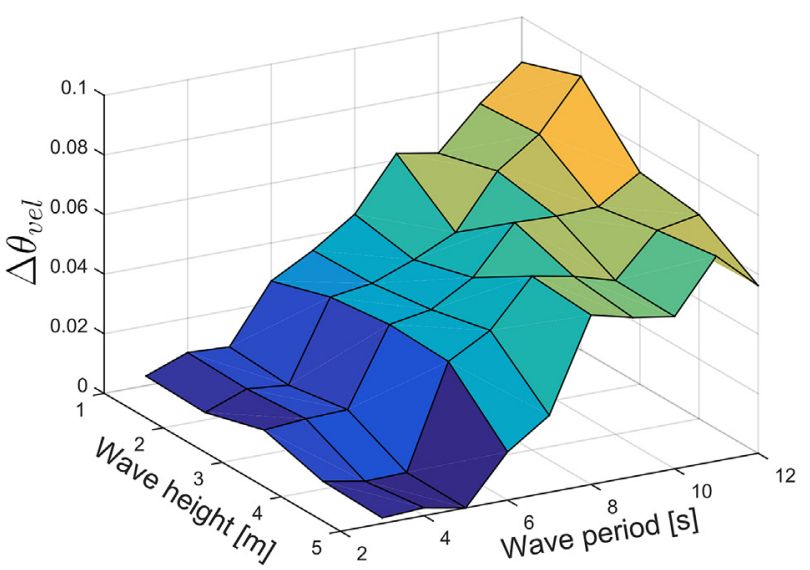

Fig. 5. The difference between $\theta_{\text {vel }}$ under ResC and ReacC.

(Faedo et al., 2017). In addition, Fig. 3 (a) suggests that the position nonlinearity is affine, which can be relatively simple to tackle from a control perspective. Nonlinearities in the power signal are also important, since a precise description of the generated power is required to accurately maximise energy generation of WECs.

Therefore, despite the lower nonlinearity measures obtained for velocity and power, the present paper especially focuses on the velocity and power nonlinearities due to their stronger implications for the

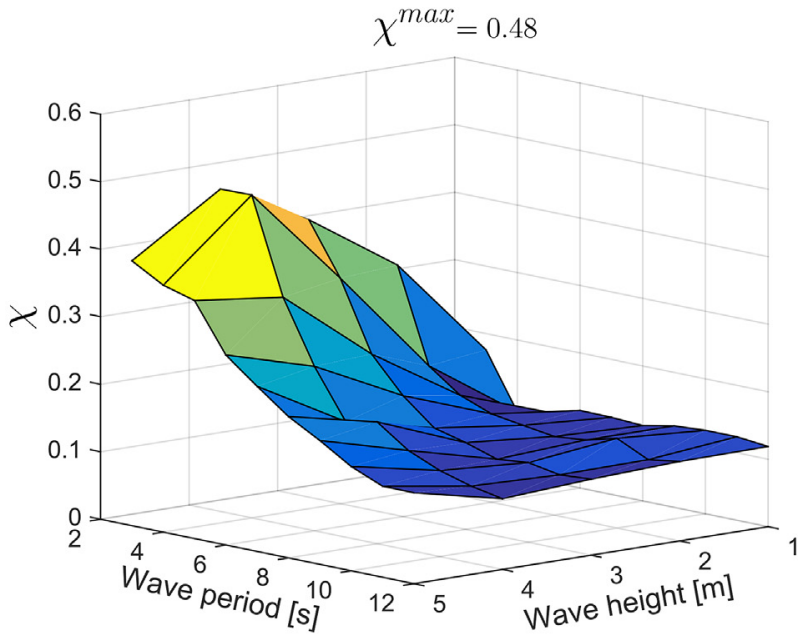

(a)

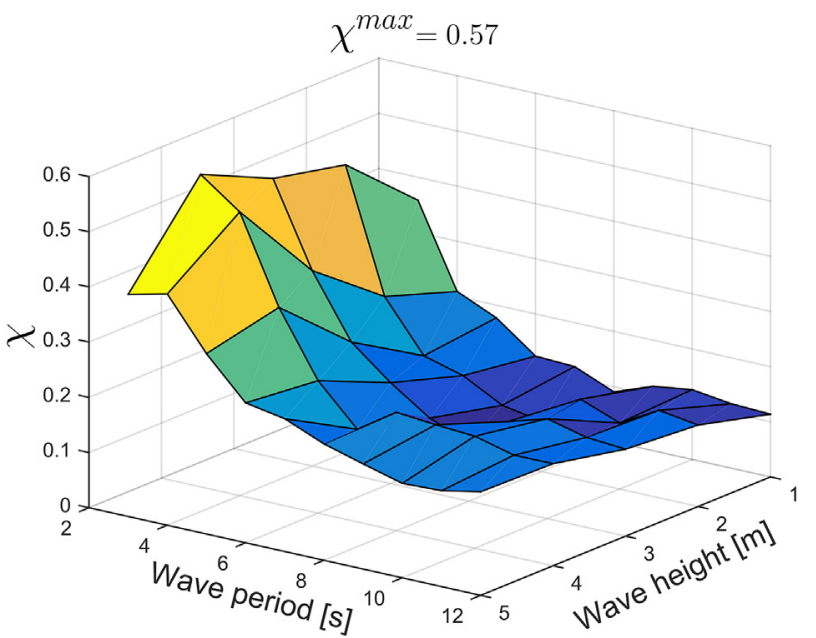

(b)

Figure 6. $\chi$ over the whole operational space for (a) the NLHyd and (b) the $N L W 2 W$ models under ReacC.

Table 1

Maximum and mean nonlinearity measures for the NLHyd and NLW2W models when using different control strategies and outputs.

\begin{tabular}{llllll}
\hline \multirow{2}{*}{ Monochromatic waves } & \multicolumn{2}{l}{ NLHyd } & \multicolumn{2}{l}{ NLW2W } \\
\cline { 3 - 6 } & & $\max$ & mean & $\max$ & mean \\
\hline \multirow{2}{*}{ ResC } & $\mathrm{Q}$ & 0.61 & 0.14 & 0.62 & 0.15 \\
& $\theta_{\text {vel }}$ & 0.33 & 0.10 & 0.36 & 0.10 \\
\multirow{2}{*}{ ReacC } & $\chi$ & 0.46 & 0.15 & 0.51 & 0.17 \\
& $\theta_{\text {pos }}$ & 0.66 & 0.16 & 0.69 & 0.20 \\
& $\theta_{\text {vel }}$ & 0.34 & 0.13 & 0.37 & 0.14 \\
& $\chi$ & 0.48 & 0.19 & 0.57 & 0.23 \\
\hline
\end{tabular}

design of energy maximising control strategies for WECs.

The NLHyd model is first studied under ResC and ReacC over the whole operational space using $\theta_{\text {vel }}$, as illustrated in Fig. 4 (a) and (b), respectively. The maximum $\theta_{\text {vel }}$ is very similar in both cases $\left(\theta_{v e l}^{\max } \sim 0.33\right)$ and is given for the same input wave (4s period and $4 \mathrm{~m}$ height). In addition, $\theta_{v e l}$ is highest with waves of $4 \mathrm{~s}$ period for all wave heights, which suggests that the natural period of the device $(3.17 \mathrm{~s}$ using fully linear hydrodynamics) increases slightly due to the nonlinear effects in the wave-absorber interaction, as previously reported in (Giorgi and Ringwood, 2016b).

However, $\theta_{\text {vel }}$ decreases substantially when moving away from the 


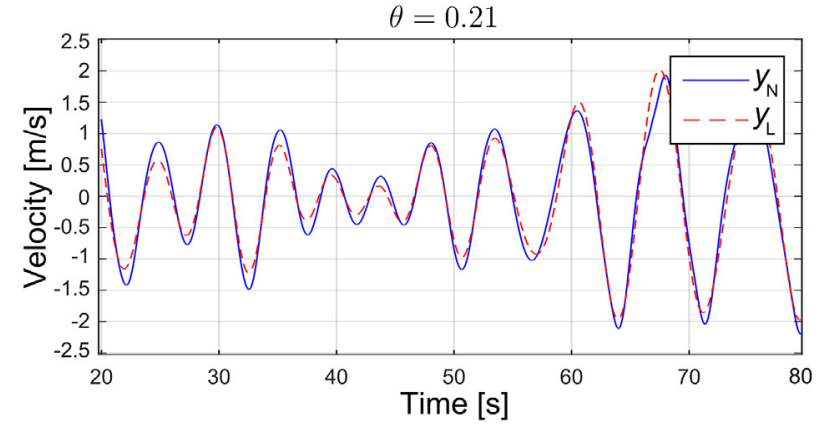

Fig. 7. The best linear approximation and $\theta_{\text {vel }}$ for a polychromatic input wave $\left(T_{p}=8 \mathrm{~s}\right.$ and $\left.H_{s}=3 \mathrm{~m}\right)$ under ResC.

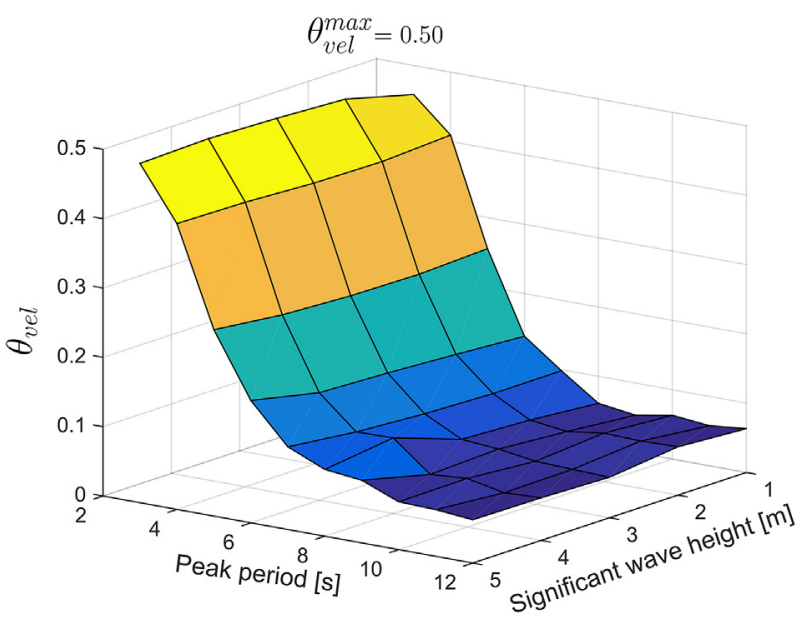

Fig. 8. $\theta_{\text {vel }}$ over the operational space for the NLHyd model under ResC and polychromatic waves.

Table 2

Maximum and mean nonlinearity measures for the NLHyd and NLW2W models when using different control strategies and outputs.

\begin{tabular}{llllll}
\hline \multirow{2}{*}{ Polychromatic waves } & \multicolumn{3}{c}{ NLHyd } & \multicolumn{3}{c}{ NLW2W } \\
\cline { 3 - 5 } & & $\max$ & mean & max & mean \\
\hline \multirow{2}{*}{ ResC } & $\theta_{\text {pos }}$ & 0.61 & 0.20 & 0.56 & 0.21 \\
& $\theta_{\text {vel }}$ & 0.49 & 0.18 & 0.59 & 0.22 \\
\multirow{2}{*}{ ReacC } & $\chi$ & 0.45 & 0.17 & 0.49 & 0.26 \\
& $\theta_{\text {pos }}$ & 0.63 & 0.21 & 0.62 & 0.21 \\
& $\theta_{\text {vel }}$ & 0.52 & 0.19 & 0.56 & 0.21 \\
& $\chi$ & 0.61 & 0.24 & 0.68 & 0.29 \\
\hline
\end{tabular}

resonant-period. For long and small off-resonance waves, $\theta_{v e l}$ vanishes under ResC, as illustrated in Fig. 4 (a), meaning that the WEC behaves linearly under these specific conditions. In contrast, $\theta_{\text {vel }}$ never vanishes in the case of ReacC, always staying above 0.1, as shown in Fig. 4 (b). Differences between ResC and ReacC are illustrated in Fig. 5, showing that $\theta_{v e l}$ significantly increases far from the resonant-period due to ReacC.

One can also note, in Fig. 4, that $\theta_{v e l}$ increases with wave height, which is to be expected, since higher waves imply greater motion and, as a consequence, greater variations of the instantaneous cross-sectional area. However, $\theta_{\text {vel }}$ is, in general, largest with wave inputs of $4 \mathrm{~m}$ height, while slightly decreasing for waves of $5 \mathrm{~m}$. This is due to the specific characteristics of the spherical WEC selected for the analysis, which has a draft of $2.5 \mathrm{~m}$. Thus, the device can exit the water with an input wave of $5 \mathrm{~m}$ height ( $2.5 \mathrm{~m}$ amplitude), reducing the power absorption from ocean waves. Therefore, a higher $B_{P T O}$ is required to

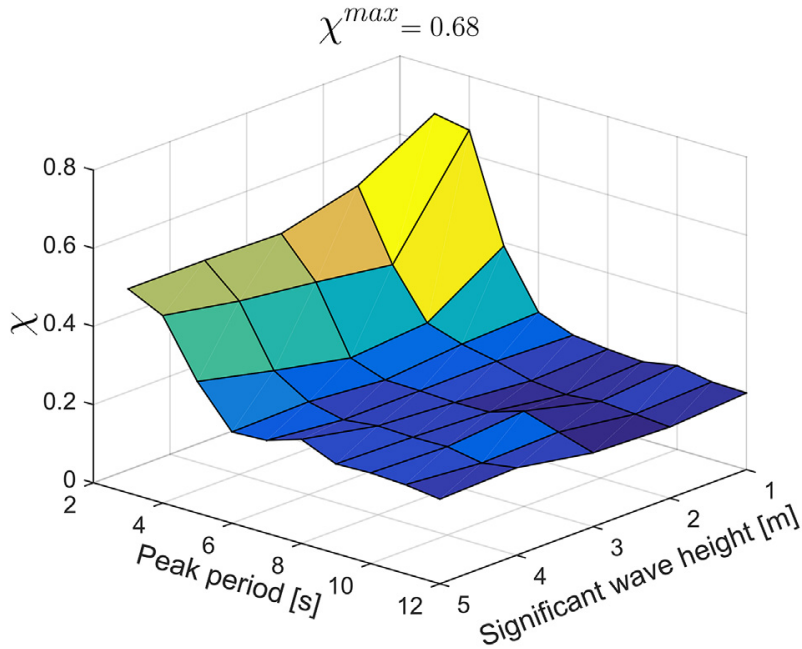

Figure 9. $\chi$ over the operational space for the $N L W 2 W$ model under ReacC and polychromatic waves.

maximise power absorption, reducing the nonlinear behaviour of the WEC.

Similar trends can be observed using power as output, as illustrated in Fig. 6 (a) and (b) for the NLHyd and NLW2W models under ReacC, respectively. While $\theta_{v e l}^{\max }$ remains similar for all the cases, $\chi^{\max }$ increases considerably, between 10 and $20 \%$, when nonlinear dynamics of the PTO are included, as illustrated in Fig. 6. Table 1 presents the maximum nonlinearity measures for all the different cases using monochromatic waves.

However, maximum nonlinearity measures tend to describe the behaviour of the WEC close to resonance, while the main differences between the different controllers and models are observed far from the resonant-period, as shown in Figs. 4-6. To illustrate an overall nonlinearity measure, mean values of the nonlinearity measures over the whole operational space are also considered. Table 1 shows these mean values, which are all similar under ResC, regardless of the model. Conversely, mean nonlinearity measures increase up to $25 \%$ from ResC to ReacC, where the highest mean nonlinearity measure is given by the $N L W 2 W$ model under ReacC $\left(\chi^{\text {mean }}=0.23\right)$.

Nevertheless, real ocean waves are never monochromatic and, therefore, the same analysis is also carried out for polychromatic waves, based on the JONSWAP spectrum (Hasselmann, 1973). The same nonlinearity measures can be applied with polychromatic waves, but the best linear approximation is parametrized using 5 basis functions with appropriately distributed time constants between $0.1 \mathrm{~s}$ and $16 \mathrm{~s}$. Fig. 7 illustrates the best linear approximation and $\theta_{\text {vel }}$ for the NLHyd model using a polychromatic input wave of $8 \mathrm{~s}$ peak period $\left(T_{p}\right)$ and $2 \mathrm{~m}$ significant wave height $\left(H_{s}\right)$ under ResC.

In the case of polychromatic waves, the operational space is defined using peak periods and significant wave heights. Fig. 8 illustrates $\theta_{\text {vel }}$ for the NLHyd model under ResC over the whole operational space.

Compared to the same case with monochromatic waves, illustrated in Fig. 4 (a), there exist three main differences. Firstly, $\theta_{v e l}^{\max }$ is substantially larger in polychromatic waves, approximately $40 \%$ higher. Second, although $\theta_{v e l}$ decreases when moving away from the resonantperiod, the nonlinear behaviour of the WEC never vanishes completely as in the case with monochromatic input waves. Finally, $\theta_{\text {vel }}$ varies very little with wave height.

Maximum and mean nonlinearity measures for each case using polychromatic waves are presented in Table 2. Mean nonlinearity measures increase significantly (up to $30 \%$ ) from monochromatic to polychromatic input waves, meaning that nonlinear effects are more significant in polychromatic waves. Measures $\theta_{v e l}^{\max }$ and $\chi^{\max }$ also increase significantly for all the cases, while $\theta_{\text {pos }}^{\max }$ remains relatively 


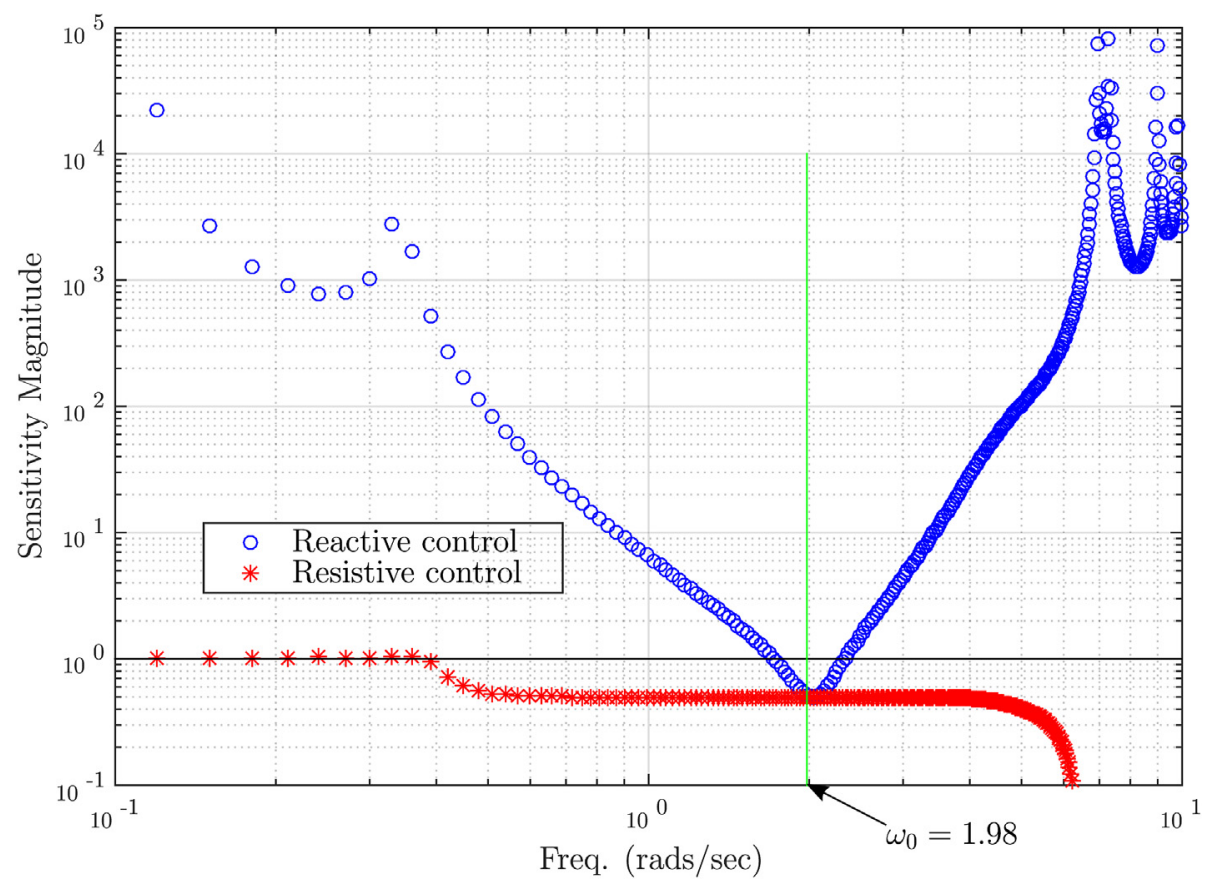

Fig. 10. The system sensitivity function, under resistive and reactive control.

unchanged.

Nonlinear effects are strongest in polychromatic waves when ReacC is implemented in the $N L W 2 W$ model, similarly to monochromatic waves. Fig. 9 illustrates $\chi$ over the whole operational space when ReacC is implemented in the $N L W 2 W$ model, where $\chi^{\max }$ unexpectedly appears at $T_{p}=3 s$ and $H_{s}=1 \mathrm{~m}$. At such a low energetic sea-state, the power absorbed by the WEC can hardly overcome the high inertia of the electric generator shaft. Therefore, the electric generator operates as an electric motor most of the time, consuming energy from the electricity grid even with appropriately optimized control parameters (Penalba and Ringwood, 2018), which results in a highly nonlinear response.

\section{Conclusion}

The paper evaluates a popular linearisation-based nonlinearity measure, the original nonlinearity measure, and shows its effectiveness in the wave energy application domain. An important modification is made so that the calculation of the nonlinearity measure itself does not include nonlinearity for power calculations. Therefore, the adapted power nonlinearity measure may be valuable in other power system applications.

Specifically, from the results presented in the paper, we can conclude the following:

- Both original and power nonlinearity measures increase for operation around the resonant period of the device. This is due to an increase in nonlinear Froude-Krylov forces resulting from larger variations in the cross-sectional area (larger displacements relative to the freesurface elevation). In addition, velocity-dependent nonlinearities, such as viscous losses in the hydrodynamic model and increased friction effects in the hydraulic cylinder, are enhanced around the resonance points.

- For the model that combines nonlinear hydrodynamic interactions with a high-fidelity power take-off, there is an increase in the power nonlinearity measure by $20 \%$ over the case for the hydrodynamic model only, over the full operational space, indicating that the primary nonlinearity in the system is hydrodynamic.

- Due to its increased aggressiveness, the reactive controller elicits a greater nonlinear response than the resistive controller, due to only the nonlinearities in the wave energy converter model, again related to increases in the wave energy converter displacement and velocity, for the reactive control case. This may suggest that, under reactive control, a nonlinear wave energy converter model may need to be considered in the control design process. The same can be applied to other type of control strategies, such as latching control, where the motion of the wave energy converter is enhanced to maximise energy absorption from ocean waves. This is especially pertinent, since the system sensitivity function (i.e. the sensitivity of the closed-loop system, containing both wave energy converter and controller, to errors in the wave energy converter model) is large for the reactive control case.

In particular, the system sensitivity, calculated using a linearised wave energy converter model $(G(s)$ ), is defined (Ringwood et al., ) as follows:

$S_{G}^{T}(s)=\frac{d T(s)}{d G(s)} \frac{G(s)}{T(s)}=\frac{1}{1+G(s) H(s)}$.

where $T(s)$ is the closed-loop transfer function, and $H_{s}$ describes the wave energy converter controller transfer function, as shown in Fig. 1, defined in time-domain form in Equation (10). Fig. 10 illustrates an example sensitivity function, comparing resistive and reactive control, and includes the block-diagram of the closed-loop system of a wave energy converter. The large sensitivity values for the reactive control, particularly off-resonance, indicate the need for an accurate model.

\section{Acknowledgment}

This material is based upon works supported by Science Foundation Ireland under Grant No. 13/IA/1886.

\section{References}

Allgöwer, F., 1995. Definition and computation of a nonlinearity measure. IFAC Proceedings 28 (14), 257-262.

Babarit, A., Delhommeau, G., 2015. Theoretical and numerical aspects of the open source BEM solver NEMOH. In: Proceedings of the 11th European Wave and Tidal Energy Conference, Nantes, France.

Babarit, A., Mouslim, H., Clément, A., Laporte-Weywada, P., 2009. On the numerical 
modelling of the nonlinear behaviour of a wave energy converter. In: Proceedings of the 28th International Conference on Offshore Mechanics \& Arctic Engineering. OMAE, Honolulu, Hawaii.

Bacelli, G., Ringwood, J.V., 2015. Numerical optimal control of wave energy converters. IEEE Trans. Sustain. Energy. 6 (2), 294-302.

Bacelli, G., Genest, R., Ringwood, J.V., 2015. Nonlinear control of flap-type wave energy converter with a non-ideal power take-off system. Annu. Rev. Contr. 40, 116-126. https://doi.org/10.1016/j.arcontrol.2015.09.006.

Boyd, S., Vandenberghe, L., 2004. Convex Optimization. Cambridge university press.

Carter, G.C., 1987. Coherence and time delay estimation. Proc. IEEE 75 (2), 236-255.

Cheng, D., Hu, X., Shen, T., 2010. Linearization of nonlinear systems. In: Analysis and Design of Nonlinear Control Systems. Springer, pp. 279-313.

Choudhury, M.S., Shah, S.L., Thornhill, N.F., 2004. Diagnosis of poor control-loop performance using higher-order statistics. Automatica 40 (10), 1719-1728.

Choudhury, A.A.S., Shah, S.L., Thornhill, N.F., 2008. Diagnosis of Process Nonlinearities and Valve Stiction: Data Driven Approaches. Springer Science \& Business Media.

Davidson, J., Giorgi, S., Ringwood, J.V., 2015a. Linear parametric hydrodynamic models for ocean wave energy converters identified from numerical wave tank experiments. Ocean Eng. 103, 31-39. https://doi.org/10.1016/j.oceaneng.2015.04.056.

Davidson, J., Giorgi, S., Ringwood, J., 2015b. Numerical Wave Tank Identification of Nonlinear Discrete Time Hydrodynamic Models. Renewable Energies Offshore, pp. 279.

Desoer, C., Wang, Y.-T., 1980. Foundations of feedback theory for nonlinear dynamical systems. IEEE Trans. Circ. Syst. 27 (2), 104-123. https://doi.org/10.1109/TCS.1980. 1084787.

Emara-Shabaik, H.E., Bomberger, J., Seborg, D.E., 1996. Cumulant/bispectrum model structure identification applied to a ph neutralization process. In: Control'96, UKACC International Conference on Control, vol. 2. IET, Exeter, UK, pp. 1046-1051.

Faedo, N., Olaya, S., Ringwood, J.V., 2017. Optimal control, mpc and mpc-like algorithms for wave energy systems: an overview. IFAC J. Syst. Contr. 1, 37-56. https://doi.org/ 10.1016/j.ifacsc.2017.07.001.

Faedo, N., Scarciotti, G., Astolfi, A., Ringwood, J., 2018. Energy-maximising control of wave energy devices using a moment-domain representation. Contr. Eng. Pract. 81, 85-96. https://doi.org/10.1016/j.conengprac.2018.08.010.

Faedo, N., Pena-Sanchez, Y., Ringwood, J., 2018b. Finite-order hydrodynamic model determination for wave energy applications using moment-matching. Ocean Eng. 163, 251-263. https://doi.org/10.1016/j.oceaneng.2018.05.037.

Giorgi, S., 2017. Linear and Nonlinear Parametric Hydrodynamic Models for Wave Energy Converters Identified from Recorded Data. Ph.D. thesis. Electronic Engineering Department, Maynooth University.

Giorgi, G., Ringwood, J.V., 2016a. Computationally efficient nonlinear froude-krylov force calculations for heaving axisymmetric wave energy point absorbers. J. Ocean Eng. and Mar Eng. 1-13.

Giorgi, G., Ringwood, J.V., 2016b. Implementation of latching control in a numerical wave tank with regular waves. J. Ocean Eng. 2 (2), 211-226.

Giorgi, G., Penalba, M., Ringwood, J.V., 2016. Nonlinear hydrodynamic force relevance for different wave energy converter types. In: Proceedings of the 3rd Asian Wave and Tidal Energy Conference, Singapour, pp. 154-162.

Guay, M., McLellan, P., 1997. Measurement of dynamic process nonlinearity. IFAC Proceedings Volumes 30 (9), 589-594.

Guay, M., McLellan, P., Bacon, D., 1995. Measurement of nonlinearity in chemical process control systems: the steady state map. Can. J. Chem. Eng. 73 (6), 868-882.

Haber, R., 1985. Nonlinearity tests for dynamic processes. IFAC Syposium on Identification and System Parameter Estimation 18 (5), 409-413. https://doi.org/10. 1016/S1474-6670(17)60594-9.
Hasselmann, K., 1973. Measurements of wind wave growth and swell decay during the joint north sea wave project (jonswap). Dtsch. Hydrogr. Z. 8, 95.

Helbig, A., Marquardt, W., Allgöwer, F., 2000. Nonlinearity measures: definition, computation and applications. J. Process Contr. 10 (2), 113-123.

Jelali, M., Kroll, A., 2012. Hydraulic Servo-systems: Modelling, Identification and Control. Springer Science \& Business Media.

Krause, P.C., Wasynczuk, O., Sudhoff, S.D., Pekarek, S., 2013. Analysis of Electric Machinery and Drive Systems, third ed. IEEE Press Series on Power Engineering, Wiley-Blackwell.

Li, G., 2017. Nonlinear model predictive control of a wave energy converter based on differential flatness parameterisation. Int. J. Contr. 90 (1), 68-77.

J. R. Morison, M. P. O'Brien, J. W. Johnson, S. A. Schaaf, The forces exerted by surface waves on pliles, Petroleum Trans., AIME. Vol. 189, pp. 149-157.

Mork, G., Barstow, S., Kabuth, A., Pontes, M.T., 2010. Assessing the global wave energy potential, 29th International Conference on Ocean. Offshore and Arctic Engineering 447-454. https://doi.org/10.1115/OMAE2010-20473.

Nikolaou, M., 1993. When is nonlinear dynamic modeling necessary? In: American Control Conference. IEEE, San Francisco, California, pp. 910-914.

Nikolaou, M., Hanagandi, V., 1994. The 2-norm for nonlinear processes: application to modeling and control problems. In: Proc. PSE'94, Kyongju, Korea, pp. 971-976.

Penalba, M., Ringwood, J., 2019. A complete validated wave-to-wire multi-rate computational model for wave energy converters,. Renew. Energy 134, 367-378. https:// doi.org/10.1016/j.renene.2018.11.040.

Penalba, M., Ringwood, J., 2018. The impact of wave-to-wire models in control parameter optimisation and power assessment. In: Proceedings of the 37th International Conference on Ocean, Offshore and Artic Engineering, OMAE,No. 77501, Madrid, Spain.

Penalba, M., Mérigaud, A., Gilloteaux, J.-C., Ringwood, J.V., 2017d. Influence of nonlinear froude-krylov forces on the performance of two wave energy point absorbers J. Ocean Eng. Mar. Energy 3 (3), 209-220. https://doi.org/10.1007/s40722-0170082-x.

Penalba, M., Giorgi, G., Ringwood, J.V., 2017a. Mathematical modelling of wave energy converters: a review of nonlinear approaches. Renew. Sustain. Energy Rev. 78, 1188-1207. https://doi.org/10.1016/j.rser.2016.11.137.

Penalba, M., Sell, N., Hillis, A., Ringwood, J., 2017b. Validating a wave-to-wire model for a wave energy converterpart i: the hydraulic transmission system. Energies 10 (7), 977. https://doi.org/10.3390/en10070977.

Penalba, M., Touzón, I., Lopez-Mendia, J., Nava, V., 2017c. A numerical study on the hydrodynamic impact of device slenderness and array size in wave energy farms in realistic wave climates. Ocean Eng. 142, 224-232. https://doi.org/10.1016/j. oceaneng.2017.06.047.

J. V. Ringwood, A. Mérigaud, N. Faedo, F. Fusco, On the Sensitivity and Robustness of Wave Energy Control Systems, (Submitted to IEEE Transactions on Control Systems Technology).

M. Ruel, J. Gerry, Quebec Quandary Solved by Fourier Transform, PULP AND PAPER

Schlöesser, W., 1961. Mathematical Model for Displacement Pumps and Motors, Hydraulic Power Transmission. pp. 252-257.

Schlösser, W., 1968. The overall efficiency of positive-displacement pumps. In: In BHRA Fluid Power Symposium, pp. 34-48.

Tarrant, K., Meskell, C., 2016. Investigation on parametrically excited motions of point absorbers in regular waves. Ocean Eng. 111, 67-81.

Theiler, J., Eubank, S., Longtin, A., Galdrikian, B., Farmer, J.D., 1992. Testing for nonlinearity in time series: the method of surrogate data. Phys. Nonlinear Phenom. 58 (1-4), 77-94. 